\title{
Water circulation induced by mechanical aerators in a rectangular vessel for shrimp aquaculture
}

\author{
Tomoaki Itano $^{\mathrm{a}, *}$, Taishi Inagaki ${ }^{\mathrm{a}}$, Choji Nakamura ${ }^{\mathrm{a}}$, Ren Hashimoto ${ }^{\mathrm{a}}$, \\ Naohiro Negoro ${ }^{a}$, Jinsuke Hyodo ${ }^{b}$, Syuta Honda ${ }^{a}$ \\ ${ }^{a}$ Department of Pure and Applied Physics, Faculty of System Engineering, Kansai \\ university \\ ${ }^{b} M M A$ Solutions Co. Ltd.
}

\begin{abstract}
A water streamer was designed for the purpose of enhancing cost-efficient circulative flow between the water surface and bottom in shrimp aquaculture ponds. We took direct measurements of the water current field induced by the designed aerator in a large rectangular reservoir of dimensions $(\mathrm{L}) 50 \times(\mathrm{W}) 19 \times(\mathrm{D}) 1.3 \mathrm{~m}^{3}$ and compared the results with those of a standard Taiwanese paddle-wheel aerator. Vertical circulation between the surface and the bottom induced by the paddle wheel aerator was smaller than that by the designed aerator. Furthermore, the paddle-wheel aerator consumed more electric power than the designed aerator. The structures of water current induced by the two aerators are elucidated, and the advantages and disadvantages of the aerators are discussed. Overall, the designed aerator was found to be more efficient than the standard aerator.
\end{abstract}

Keywords: inland aquaculture, paddle-wheel aerator, circulation, water current field, dissolved oxygen, turbulent mixing

\section{Introduction}

Shrimp farming vs catches. - Aquaculture has become an important source of food and commercial products in the world today. On behalf of the Food and Agriculture Organization of the United Nations, Gillet (2008)

\footnotetext{
*Corresponding author

Email address: itano@kansai-u.ac.jp (Tomoaki Itano )
} 
reported in a comprehensive technical paper that, "Today, world annual production of shrimp, both capture and farmed, is about 6 million tonnes. Currently, over 40 percent of world shrimp production is from farming ..." In general, aquaculture is classified into marine and inland aquaculture, of which the latter is mainly employed for shrimp aquaculture. In fact, inland aquaculture (farming) is flourishing, especially in tropical and semi-tropical areas in the Far-east, Southeast Asia, and Latin America. Inland aquaculture has been supporting the recently rising global consumption of shrimp and prawns, instead of wild shrimp captures by means of trawling fisheries, the ratio of which in the total production has fallen since 1990. While shrimp aquaculture has brought about increased income for developing countries in the abovementioned areas, it has unfortunately been accompanied by the deforestation of natural mangroves for the construction of shrimp ponds and the salination of groundwater and agricultural land (Murai (2007)). Demolishing mangrove forests, which serve as nurseries of post-larval shrimp and other creatures, is likely to decrease near-shore fish and shrimp catches. Uncontrolled pursuit of commercial profits from shrimp aquaculture sometimes lead to inadequate wastewater treatment, resulting in eutrophication. As a result, residential areas surrounding aquaculture ponds may face environmental pollution. Thus, many practical issues regarding the ecological and economical sustainability of shrimp aquaculture still remain unsolved.

Aeration. - Unlike marine aquaculture in a finite space surrounded by an open environment, such as rivers or seas, inland aquaculture is operated in an isolated vessel or coastal pond with little connection to the outer environment. In such an isolated space, the biological condition spontaneously tends to extremely differ from the natural environment. Thus, the shortage of dissolved oxygen (DO) concentration in water for aquatic creatures to breathe is one of the technical issues regarding the biological water condition in inland aquaculture for shrimp. Intensive methods are commonly practiced in inland aquaculture, where the shrimp concentration is extraordinarily dense, compared to the natural density. In most cases, additional enforcement of water circulation is required in order to increase DO in water, which is called "aeration". Improved aeration can ensure commercial and eco-friendly production of shrimp through aquaculture, as well as increase the production capacity of aquaculture ponds in cases where DO is a vital factor. Moreover, an excellent aeration system is expected to induce an optimum and sustainable state in aquaculture ponds, such as "aquaponics", 
which implies a sustainable synthetic food chain in an isolated aquacultural system involving bacteria, algae, aquatic plants as well as aquatic animals.

Empirical Research. - Research and innovations in the last decades have provided fundamental understanding of aeration and improved the primitive aeration system to be capable of producing strong currents with high oxygen transfer efficiencies, thus increasing aquacultural productivity. However, aquaculture has been inherently associated with traditional methodologies, which have been improved gradually and empirically thus far. Thus modern aeration systems are still not the focus for achieving optimum conditions, and the importance of improving the aeration system is probably not understood sufficiently by most aquacultural farmers and equipment manufacturers, as pointed out by Lawson (1997). The difficulty is also attributed partly to the fact that the aquacultural aeration system is influenced by chemical, biological, and meteorological factors with less-repeatability, which is critical for scientific investigations. Here, we speculate the process of atmospheric oxygen being dissolved and delivered to aquatic creatures at the bottom, from the viewpoint of fluid dynamics.

Diffusion and Advection. - If a water body is in equilibrium with atmospheric oxygen at the surface in contact with air, there is no net transfer of oxygen between the atmosphere and water. However, when water is undersaturated with oxygen, oxygen from air will dissolve in water, and the reverse is true when water is supersaturated with oxygen. The atmospheric solubility of oxygen is mainly determined by atmospheric temperature, pressure, and gas composition, where decrease in temperature and increase in atmospheric pressure cause a corresponding increase in the amount of oxygen dissolvable in a unit volume of water. The increase of DO is also caused by increase of air-water contact interface and exposure time, which may be artificially controlled by employing mechanical aerators. Mechanical aerators splash a large amount of water near the surface into the air while releasing air bubbles under water. In this manner, they may produce foamy wave crests with air entrainment and microscopic splashes at the water surface around the aerators, significantly increasing net air-water contact interface. The instantaneous pressure may overcome the saturated vapor pressure to enhance DO locally. Even if water is macroscopically at rest, the oxygen dissolved at the surface may be diffused towards the bottom by thermal molecular motion. This process may be termed as "oxygen diffusion" from the surface to the bottom. 
Here, we should note that aquacultural shrimps do not have any swim bladder, and they mostly crawl on the pond bottom using their legs and tail. Therefore, their thriving environment may be considered a two-dimensional surface at the bottom and not a three-dimensional volumetric space. Thus, in aquaculture ponds for shrimp, the consumption of oxygen occurs mainly at the bottom, and mechanical aerators for aquaculture are required to deliver large amounts of water containing high DO concentrations from the surface to the bottom. The delivery can be performed through water circulation between the surface and the bottom and can be largely enhanced by turbulent motion with swirling motions. This process may be termed as "oxygen advection" from the surface to the bottom in the pond, the timescale of which is fairly shorter than the diffusion process.

Production, Consumption, and Realization of Equilibrium. - It should be emphasized that algae have a large influence on the amount of DO in aquacultural ponds because they produce oxygen during the daytime and consume oxygen at night. Additionally, they develop volumetrically in the pond and increase DO, but they also suppress vertical water circulation for oxygen advection. If oxygen production and consumption by algae or shrimps are absent, the distribution of DO probably remains unchanged to a large extent after saturation. Oxygen transfer in aquaculture ponds involves various physical and biological processes: dissolution, diffusion, advection, production, and consumption. To understand the entire oxygen transfer process in aquacultural ponds in detail, the entire region of aquacultural ponds, including shrimps and algae, should be comprehensively investigated.

DO Measurement in Karawang. - Here, we show an example of time variation of DO measured in aquacultural ponds. We performed commercial shrimp aquaculture at Aquaculture Business Center (ABC) Karawang, West Java, Indonesia. Time variation of DO was taken from a shrimp aquaculture in September 2016 during the dry season. The DO measurements were taken using optical DO meters (RINKO ID, JFE Advantech Co., Ltd, Hyogo, Japan). This optical DO meter works on the principle of the dependency of the emission and lifetime of light excited at the sensing membrane on the oxygen concentration around the membrane. Thus, such an optical DO sensor requires no minimum inflow velocity, which is advantageous against prevalent galvanic DO sensors that consume oxygen on the membrane and exhibit some dependency on flow current. Shrimp aquaculture was practiced in two similar aquacultural ponds with different types of mechanical aerators. DO 

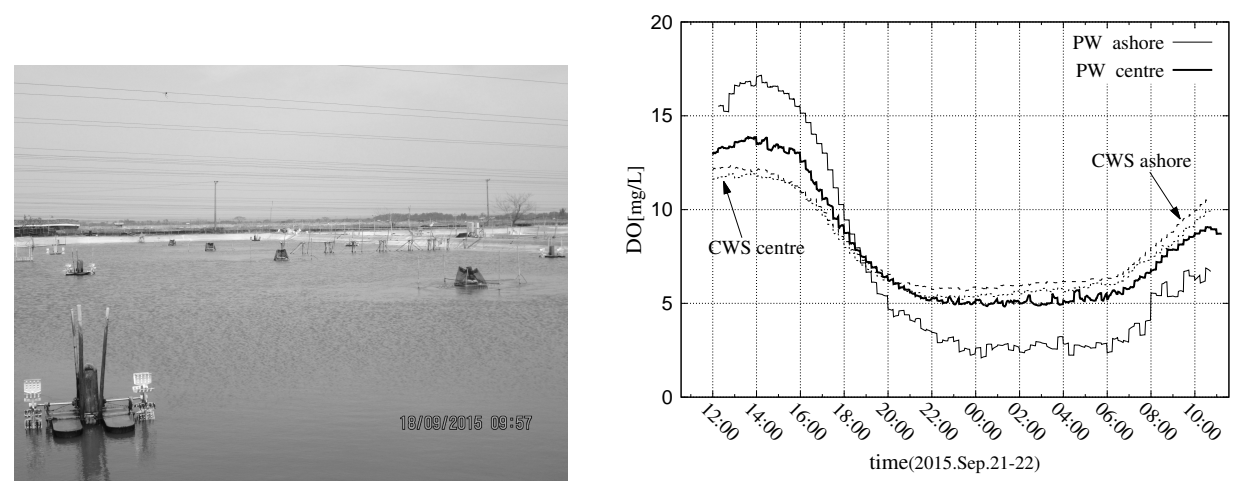

Figure 1: (left) Paddle-wheel aerators employed in an commercial shrimp pond at Karawang Indonesia in September 2016 during the dry season. (right) Time variation of DO measured in the aquacultural ponds. DO meters were set at two locations, ashore and the center of a pond; The thick curves in the figure represent the paddle-wheel (PW) pond, and dashed curve represents the centrifugal water stirrer (CWS) pond.

meters were positioned at a depth of $0.1 \mathrm{~m}$ from the water surface at two horizontal locations: one is near the center of the pond, and the other is ashore at $3 \mathrm{~m}$ from the short side of the pond of $35 \mathrm{~m} \times 135 \mathrm{~m}$. Two distinct mechanical aerators, paddle wheel $(\mathrm{PW})$ and centrifugal water stirrer (CWS), which are explained later, were placed at the center of each pond.

As shown in curves in Fig.1, all the measured DO values increased during the daytime and decreased at night. This is because oxygen produced by algae photosynthesis during daytime is consumed at night. In general, the value $2 \mathrm{mg} / \mathrm{L}$ in DO is fatal for living creatures including shrimp and one of the curves arrived at the fatal value from midnight to early morning. Note that algae has a significant influence on DO value in aquaculture ponds. Here, it should be emphasized that a couple of DO values in the CWS pond do not change largely during the day as those in the PW pond. In the daytime, DO values in CWS are less than those in the PW pond. As shown later, a paddle-wheel type of aerator works at $\mathrm{O}\left(10^{3}\right) \sim \mathrm{O}\left(10^{4}\right)$ Watts (see Cancino (2004)), which is over ten times the running cost of centrifugal water stirrer. It appears unclear as to why the DO value in the pond with circulation induced by a much cost-effective aerator is higher than that induced by the other. This is the principal motivation of the present study.

We considered that one of the reason is a difference in the distribution of water currents induced by the aerators, which contributes largely to the deliv- 
ery of DO throughout the pond. Although there have been several studies on DO concentration (Moulick et al. (2002); Cancino et al. (2004); Brown et al. (2016)), most of them are either phenomenological or macroscopic. To the best of our knowledge, previous studies are not based on the fluid mechanical viewpoint, except for a few numerical studies (Peterson et al. (2000); Pfeiffer et al. (2007); Yamayoshi et al. (2008); Huggins et al. (2004)). In the present study, we took direct measurements of the water velocity field induced by two distinct mechanical aerators located in a rectangular reservoir, using an electro-magnetic velocimeter. The structures of water current induced by the two aerators are elucidated, and the advantages and disadvantages of the aerators are discussed. Note that we ristricted ourselves in the measurement of fluid motion. The measurement of DO field, which probably requires the existence of living creatures such as shrimps at the bottom consuming some amount of oxygen delivered by aerators, will be investigated in future works.

\section{Mechanical aerators}

Paddle-wheel Aerator.- According to Lawson (1997), aquacultural aerators can be generally classified into the three categories: gravity aerators, air diffusion systems, and mechanical aerators. Furthermore, some hybrids are practically utilized. Gravity aerators employ the water potential energy as in waterfalls for the purpose of dissolving oxygen into water, whereas air diffusion systems consist of several air compressors to directly supply air into porous pipes or other devices located at a depth under water. In contrast, "mechanical aerators" include devices with either rotating vertical or hori-

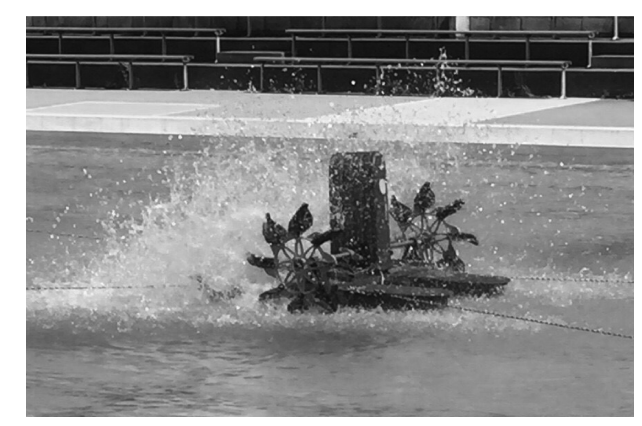

Figure 2: A paddle-wheel aerator TA-55H in action; The aerator is held by four tethers at the center of Katayama civic outdoor pool (September 2016, copied from Itano et al. $(2018))$. 
zontal shafts with paddles or screws to circulate water in the pond. Water is broken into droplets for the purpose of mixing water as well as increasing the DO concentration of water. The standard evaluation procedure of efficiencies for several types of aerators proposed by Colt and Orwicz (1991) provides evidence that, among mechanical aerators, the "paddle-wheel aerator" is relatively efficient at circulating water than other types of aerators.

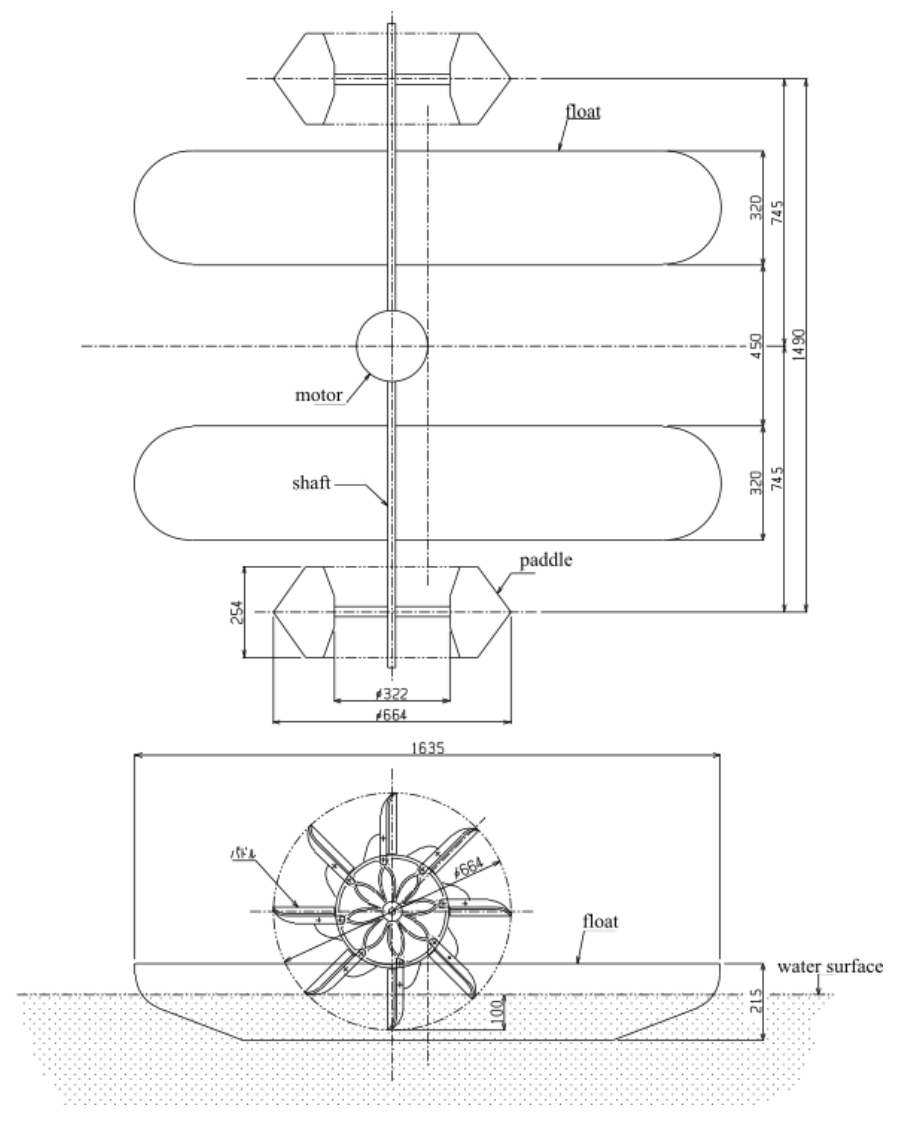

Figure 3: Plan and side views of a commercial small paddle-wheel aerator, TA-55H, manufactured by Team aqua Co. Ltd., Taiwan (copied from Itano et al. (2018)). Gray region indicates that the side view corresponds to water in the equilibrium state with the aerator floating on the water. The aerator on the pond surface produces a jet-like water stream downwards with remarkable splashing, releasing air bubbles into the water. The amount of water corresponding to the volume of the surface layer under the shaft passed by a paddle is represented as $\Delta V \mathrm{~m}^{3}$, which is approximated to $2.0 \times 10^{-3} \mathrm{~m}^{3}$.

Paddle-wheel aerators consist of a rotating horizontal shaft, "hub", driven 
by either electric motor or combustion engine, and several paddles attached on a wheel. The assembly of the hub and paddle is termed as "drum". As a sightseeing side-wheel boat plows a river, the paddles of the aerator over the pond surface produce a jet-like water downstream. Fig.2 shows a commercial small paddle-wheel aerator, TA-55H, manufactured by Team aqua Co. Ltd., Taiwan. Similar aerators have been widely adopted in aquacultural ponds to cultivate various types of fishes or shellfishes (Boyd, 1998), as well as in shrimp cultivation in Indonesia, Thailand, and Taiwan. In the present study, we installed the TA-55H aerator in a relatively large rectangular reservoir and measured the velocity field of water stream induced by the aerator.

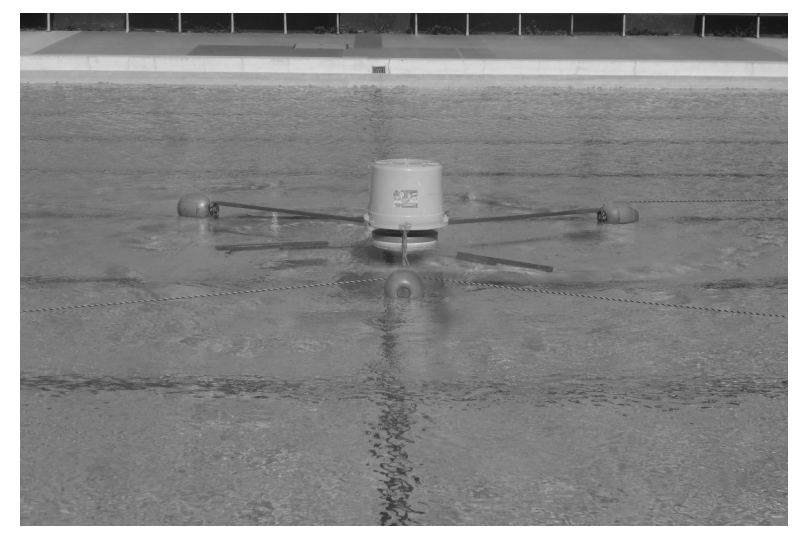

Figure 4: A centrifugal water stirrer "CWS" installed at the center of Katayama civic outdoor pool (September 2017).

The plan and side views of TA-55H are shown in Fig.3. The streamwise and spanwise extents of the aerator are $1635 \mathrm{~mm}$ and $1744 \mathrm{~mm}$, respectively. In the side view, the submerged part of the aerator (shaded, at about $100 \mathrm{~mm}$ depth) corresponds to the lower part of the float, which supports the mechanical and electrical assemblies, such as motor, reducer, and shaft, with a couple of paddle-wheels on the surface of the water. The drum can be lowered deeper into the water by adjusting the float height to increase water stream while decreasing DO in water, and vice versa. According to Lawson (1997), paddle-wheel aerators have specific optimum drum depth and rotational speed, but in practical use, most aerators operate at a paddle depth of 100 150 mm and drum rotational speed of 80 90 rpm under an electric power requirement on the order of 1000 Watts. In the present study, the motor of TA-55H was driven by alternating current with the three 


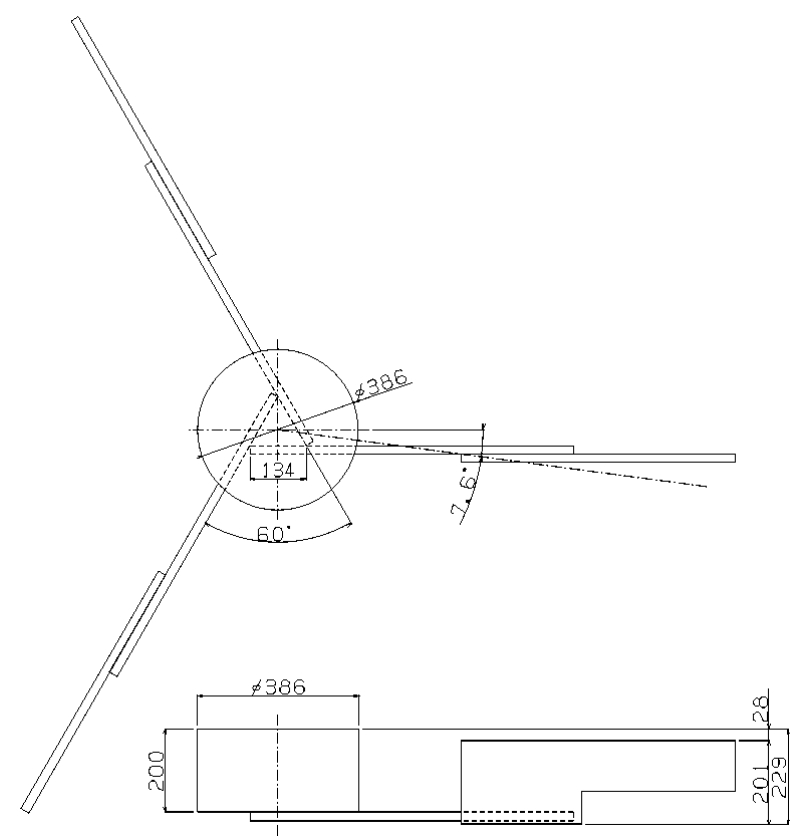

Figure 5: (upper) Top view of the centrifugal water stirrer, CWS, designed for the purpose of shrimp cultivation and water stirring in a shallow but wide pond. The original idea of this stirrer is taken from a Japanese patent provided by Inaba (2005). (lower) Side view of a paddle of the water stirrer, in the form of an inverted L. The submerged part of the paddle is shaded (copied from Inagaki et al. (2017)).

phase $200 \mathrm{~V}$ class, which was boosted by a 750 Watts class general-purpose, frequency-adjustable inverter (FR-D710W-0.1K, Mitsubishi Electric, Tokyo, Japan). The rotation rate of the main shaft could be controlled by manually adjusting the output frequency of the inverter. Via a reducer, the vertical rotation motion of motor shaft was converted to horizontal rotation motion of the shaft attached to a couple of drums. When the lower part of drums were submerged at about $100 \mathrm{~mm}$, the drums rotated at $(100 \pm 3) \mathrm{rpm}$. The distribution of water velocity in the pond, which is not directly relevant to the total volumetric flow rate of the aerator, is required to estimate the volumetric flow rate of circulation throughout the pond driven by the aerator (see Brown and Tucker (2014)).

Centrifugal Water Stirrer. - We designed another mechanical aerator, "centrifugal water stirrer" (Fig.4). The prototype of CWS is inspired by a Japanese patent provided by Inaba (2005) and practical centrifugal surface aerators (cf. Cancino et al. (2004)), which is not widely adopted in aquacul- 
tural ponds for the time being. Fig. 5 shows the top and side views of the CWS. A stainless steel paddle in the shape of an inverted L (L $545 \mathrm{~mm} \times \mathrm{W}$ $200 \mathrm{~mm}$ ), shown in the side view, is mounted on a horizontal beam (1260 $\mathrm{mm})$. The beam is attached to a vertical shaft with a sweptback angle of $7.6^{\circ}$ via a center cylindrical float $(\phi 386 \mathrm{~mm} \times \mathrm{H} 200 \mathrm{~mm})$, such that the distance between the shaft and the tip of paddle is $1190 \mathrm{~mm}$. The center cylindrical float rotates together with the shaft and the three paddles. Connected to an electric motor (3IK15A-CW2E, $1200 \mathrm{rpm}$, Oriental Motor Co., Ltd.) via a torque converter of reduction gears and pulleys with a ratio of 1:311, the shaft rotates at $T=4.3 \mathrm{rpm}$ under water resistance. The paddles rotate in the clockwise direction in the top view of the figure. Three extra floats and the main body (not illustrated in the figure) support the motor weight as well as the paddles and shaft over the water surface, and sustain the motor and the shaft in the vertical direction such that the three paddles are almost horizontal and submerged at a depth of $150 \mathrm{~mm}$.

Primarily, the paddles rotating at the surface induce azimuthal water circulation around the shaft on the horizontal plane at the surface. If the motor operate continuously, the layer of the horizontally circulating water gradually expands in the horizontal direction and thickens in the depth direction, and finally water at the bottom of the reservoir starts circulating in the bottom plane, which may form an Ekman layer at the bottom of the reservoir. Moreover, the rotation of the paddles push water away from the surface towards the radial direction due to centrifugal force partly attributable to the sweptback angle of the beams against the radial direction. Owing to these effects, water under the CWS is lifted up from the bottom, and the paddle rotation produces secondary circulation between the surface and the bottom in the reservoir. It should be noted that such circulation between the surface and the bottom in the reservoir is not expected for paddle-wheel aerators prevalent in aquaculture. The optimal volumetric flux induced by the CWS working under a constant electric power 25 Watts is estimated to be 920 $\mathrm{m}^{3} / \mathrm{h}$ based on the original patent.

\section{Measurements}

Reservoir. - The present measurement of the water velocity field induced by the aforementioned mechanical aerators was carried out at Katayama civic pool of Suita city, Osaka, Japan. The measurements were taken on several calm days in the autumns of 2016 and 2017, during which public swimming 
was on hold. The Japan meteorological agency officially provides wind speed measured every 10 min at an observatory station in Toyonaka city adjacent to Suita city. The wind speed during the measurement periods was less than $4.1 \mathrm{~m} / \mathrm{s}$ at maximum. This civic pool possesses an indoor reservoir and three outdoor reservoirs, among which the largest is an outdoor reservoir of about 0.1 ha extent $((\mathrm{L}) 50 \mathrm{~m} \times(\mathrm{W}) 19 \mathrm{~m} ; 1.3 \mathrm{~m}$ average water depth) with several tiny ducts for sluice and drainage. In the presence of the main building of the indoor reservoir and a few private residential apartments surrounding the outdoor reservoir, the background atmospheric wind is weak, variational, and non-directional. Therefore, its effect on the water stream con be considered to be negligible, which was confirmed by measurements of the water stream under calm wind with no aerator. The bottom of the reservoir was designed with a slight incline towards the center from the two short sides for the purpose of drainage. The maximum depth of water is $1.4 \mathrm{~m}$ at the center, and $1.2 \mathrm{~m}$ at the sides. In addition, there are narrow ramps of $0.4 \mathrm{~m}$ height along the two long sides at the bottom. The reservoir comprises concrete foundation and walls coated with waterproof paint and has an exactly rectangular shape from the top view (Fig.6). The center of the reservoir on the surface was taken as the origin of our Cartesian coordinate system, and the coordinates $x$ and $y$ were taken as the short and long sides, respectively, and $z$ is from the surface to the bottom.

Tethering aerators. - Although the sluice and drainage system were stopped at the beginning of September, aquatic bacteria, insects and algae did not flourish until the end of September because of chlorine, which was used to prevent them from proliferating during the public swimming service. Tethered to the four corners of the reservoir, the two mechanical aerators, TA-55H and CWS, were installed one by one (TA-55H was fixed as its jet downstream was directed along $y$ direction). The drums of TA-55H were submerged by about $100 \mathrm{~mm}$, and their rotational speed was manually maintained between 97 103 times per a minute on average, by controlling the output frequency of the inverter. The three paddles of CWS were submerged by about 150 $\mathrm{mm}$ in depth, and their rotation rate was maintained at $4.3 \mathrm{rpm}$ under water resistance.

Electro-magnetic Water Current Meter. - Velocity fields were constructed from the current vector measured at several grid points in the pool. The measurements were made using two electro-magnetic water current meters (VM-801L and VMT2-50-08PS, Kenek Co., LTD., Tokyo, Japan). The probe 


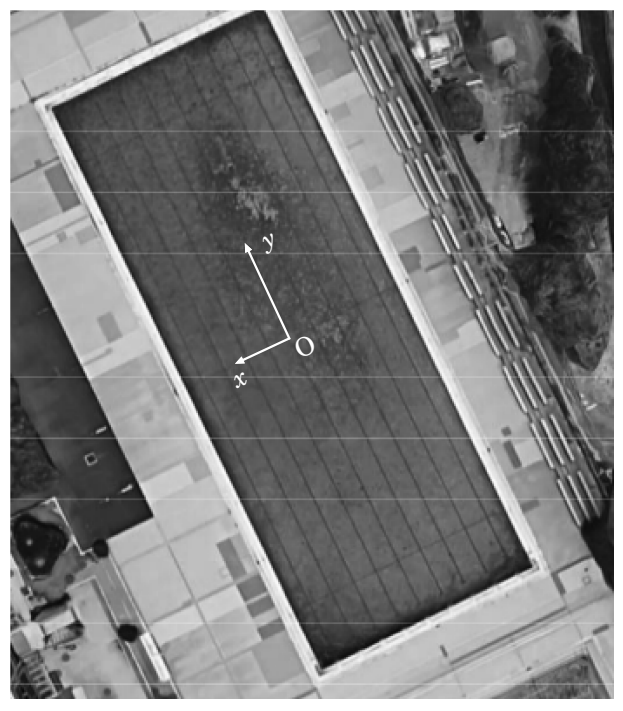

Figure 6: Satellite photograph of the largest outdoor reservoir in Katayama civic outdoor pool. The dimensions of the reservoir are $(\mathrm{L}) 50 \times(\mathrm{W}) 19 \times(\mathrm{D}) 1.3\left[\mathrm{~m}^{3}\right]$.

of this current meter is oval shaped, scaled by about $10 \mathrm{~mm}$. Normal components of the water velocity perpendicular to the major axis of the oval were continuously obtained as analog voltage outputs via amplification. We fixed the oval major axis of the probe towards the $z$ direction so as to obtain $x$ and $y$ components of water velocity of the downstream behind the paddle-wheel aerator and around the CWS. It is guaranteed by the manufacturer that the current meter provides an accuracy within $2 \%$ of the full scale selected from the ranges of 100,250 , and $500 \mathrm{~mm} / \mathrm{s}$. Thus, the current meter can distinguish water velocity up to $2 \mathrm{~mm} / \mathrm{s}$ at least, which is an advantage in identifying subtle water streams induced at a large distance from the aerator.

Average and Dispersion of Water Velocity. - For each measurement at a grid position, a time series of water velocity every second over 600 seconds was recorded using digital data logger MR8875 (Hioki E.E. Corp., Nagano, Japan). Fig.7 shows the time series of horizontal components of water velocity taken at the grid point closest to the two aerators. The variance of the time series obtained from the paddle-wheel aerator is apparently larger than that from the CWS. This can be attributed to the fact that the water current induced by CWS is more stable. In the figure, both the time series possess several characteristic frequencies. While the fastest variations common at all the measurement points could be attributed either to the ro- 

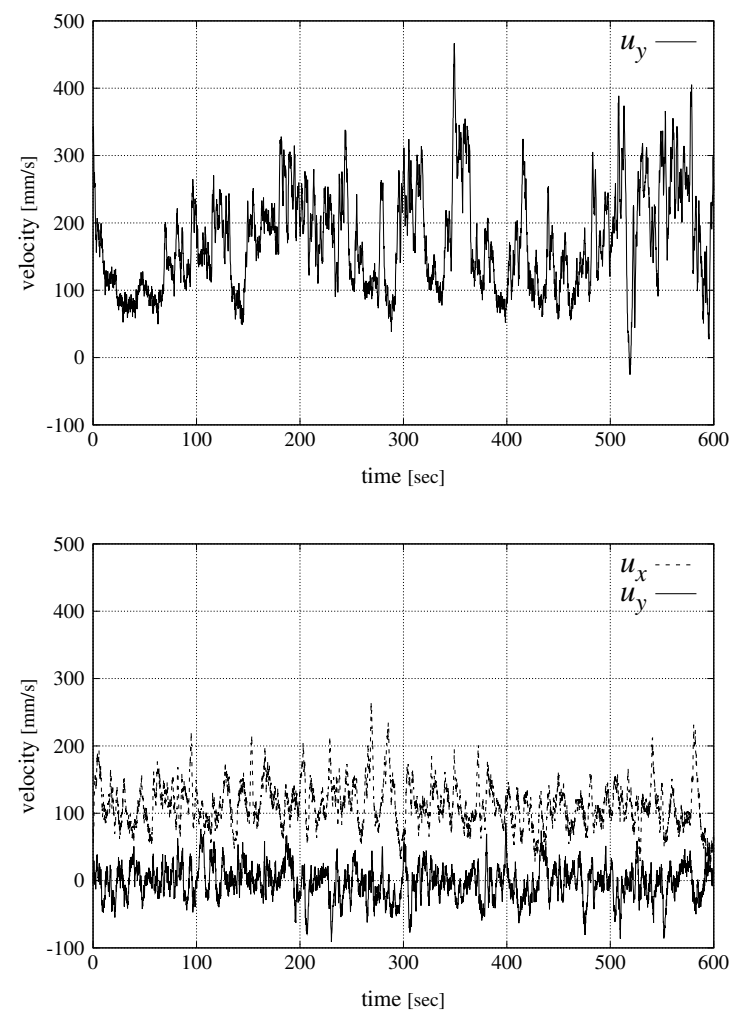

Figure 7: Raw data of the time series of horizontal components of water velocity taken at the point $(x, y, z)=(0.0 \mathrm{~m}, 3.0 \mathrm{~m}, 0.1 \mathrm{~m})$ for $600 \mathrm{~s}$. (left) Water flow induced by the paddle-wheel aerator on the 25th of October in 2017. (right) Water flow induced by the CWS on the 14th of September in 2017.

tational frequency of the drums and the paddles, the slow variation peculiar to the measurement at the grid points near the aerator is attributed to a certain unsteady large-scale turbulent motion around the aerator, which will be investigated future works. The average $\bar{u}_{i}$ and the standard deviation $\sigma\left(u_{i}\right)$ of water velocity at each grid point is calculated from the raw data, as follows:

$$
\begin{aligned}
\bar{u}_{i}(x, y, z) & =\frac{1}{T} \int_{0}^{T} u_{i}(t, x, y, z) \mathrm{d} t \\
\sigma\left(u_{i}(x, y, z)\right) & =\sqrt{\frac{1}{T} \int_{0}^{T}\left(u_{i}(t, x, y, z)-\bar{u}_{i}(x, y, z)\right) \mathrm{d} t},
\end{aligned}
$$


where $i(i=y, z)$ is the component index and $T$ is the total measurement time.

\section{Velocity Field}

Velocity Field by TA-55H. - The velocity field, $\left(\bar{u}_{x}, \bar{u}_{y}\right)$, in Fig.8 is the mean water current induced by TA-55H. The measurements were taken at several grid points at the two horizontal planes, (shallow) $z=0.1 \mathrm{~m}$ and (deep) $z=1.1 \mathrm{~m}$ downstream of the aerator. The average and variance were obtained from sequential raw data taken for $T=600 \mathrm{~s}$ per measurement at a grid point. The $x$ and $y$ error bars centered at the tip of vectors represent a fluctuation of the water current estimated from the standard deviation, $\pm \sigma\left(u_{i}(x, y, z)\right)$ for $i=x, y$.
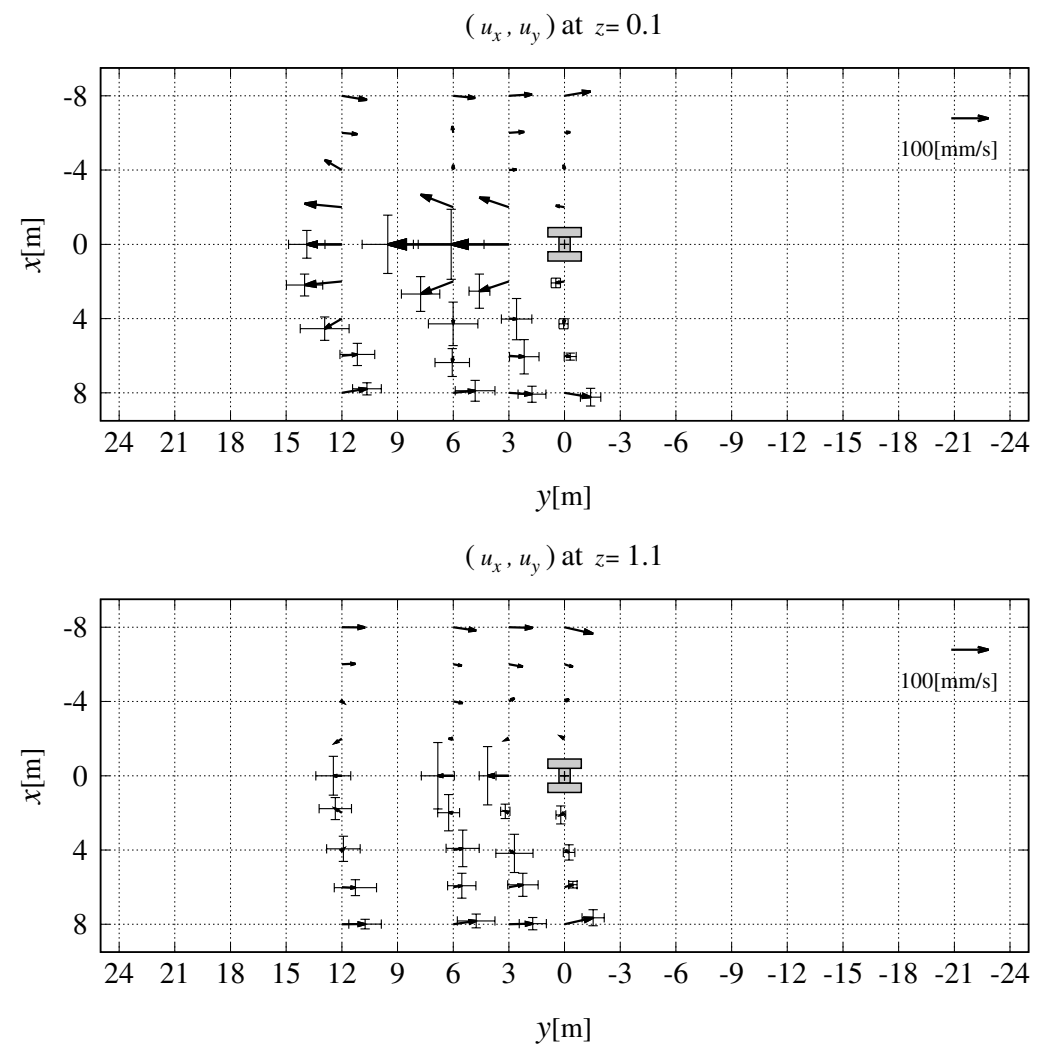

Figure 8: Velocity field of the water current induced by TA-55H. Measurements were taken at two horizontal planes, (upper) $z=0.1 \mathrm{~m}$ and (lower) $z=1.1 \mathrm{~m}$. 
As shown in the figure, TA-55H induces a unidirectional jet stream downwards along the $y$ axis from the origin. The magnitude of water current is over $200 \mathrm{~mm} / \mathrm{s}$ at the measurement grid closest to the aerator. The jet stream is dispersed and reduced at the downstream away from the aerator. Under the restriction of the reservoir wall, certain return currents were observed within $4 \mathrm{~m}$ from the side walls located at $x= \pm 9$, which would probably be weaker in a larger reservoir, such as a commercial aquacultural pond.

It should be noted that the water current at the bottom of the reservoir $(z=1.1)$ is similar to that at the surface $(z=0.1)$. This simply suggests that the water current at the bottom is induced via shear stress attributed to the surface current, which is driven primarily by the aerator. Accordingly, it is implied that the mean flow does not largely contribute to the vertical circulation expected for the purpose of mixing high DO water between the surface and the bottom of the reservoir. Such mixing in the vertical direction is dependent on unsteady turbulent flow inherent to the jet stream, which is reflected in large fluctuations near the origin, as shown in the figure.

Velocity Field by CWS on the Vertical Cross Section.- We additionally measured the mean water velocity field on the $y$-z plane, $\left(\bar{u}_{y}, \bar{u}_{z}\right)$, which is mapped in Fig.9. The error bars spanned at the tip of each vector represent the current fluctuation observed in the time series. From the figure, we may conclude that the aerator located at the origin provides a horizontal and almost unidirectional jet stream downwards throughout the reservoir under consideration. Thus, a fluid element travels on a horizontal plane at a depth in average along trajectories resembling the Arabic numeral eight. In fact, from the data obtained at several grid points at $x=0$ and $6 \mathrm{~m}<$ $y<12 \mathrm{~m}$, a downward jet-like flow, $u_{y}$, is interpolated as approximately $u_{y}=-0.014 y+0.26$. If we assume the incompressibility of water current and the mirror symmetry on the $x=0$ plane, then $\frac{\partial u_{z}}{\partial z}=-\frac{\partial u_{x}}{\partial x}=0.014 / \mathrm{s}$. Thus we obtain $u_{z}=1.4 \mathrm{~mm} / \mathrm{s}$ on the $y$ axis as a rough estimation.

Velocity Field by CWS. — The mean water velocity fields induced by CWS, $\left(\bar{u}_{x}, \bar{u}_{y}\right)$, taken at the horizontal planes at $z=0.1 \mathrm{~m}$ and $z=1.1 \mathrm{~m}$ are mapped in Fig.10. The error bars at the tip of each vector represent those in Fig.8. We note that, compared with the error bars in Fig.8, the fluctuation of water current induced by CWS is relatively small even near the aerator. The small fluctuations at all the grid points suggest that the current is relatively steady and that the water current structure estimated from the mean velocity measured is robust. 


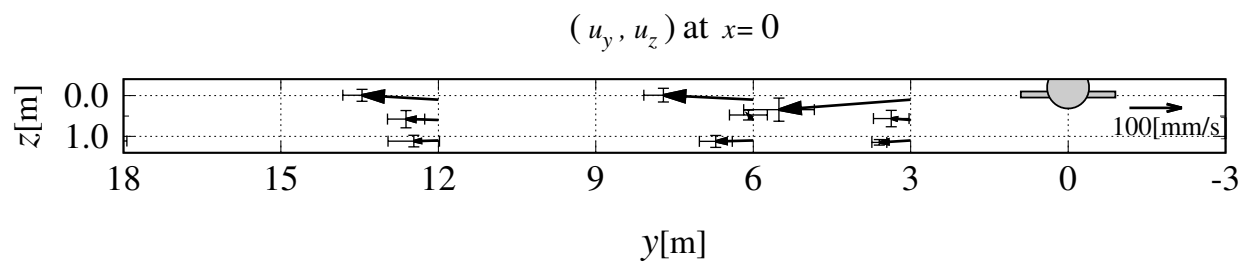

Figure 9: Velocity field of the water current induced by CWS on the $y$ - $z$ plane. Measurements were taken at three different depths, $z=0.1 \mathrm{~m}, 0.6 \mathrm{~m}, 1.1 \mathrm{~m}$.

\section{Discussion}

Note that the velocity fields at the surface and bottom planes are remarkably different, which is in contrast to Fig.8. The azimuthal component of the induced velocity fields are clockwise in both cases. Therefore, the horizontal circulation common in Fig.10 may be attributed to shear stress between the surface and bottom planes. In contrast, the radial component of water current on the surface and bottom planes are positive and negative, respectively. Taking into account that the velocity field is incompressible, we can deduce the existence of upward flow under CWS, that is, circulation between the surface and bottom of the reservoir. The expected spiral trajectories of fluid elements under CWS is illustrated in Fig.11. A passive tracer travels along one of the spiral trajectories in a cylindrical region around the $z$ axis with a radius. From the vector field in figure10, the radial component of $u_{r}$ at $z=0.1$ can be interpolated as $u_{r}=-0.01 x+0.1 \mathrm{~m} / \mathrm{s}$ at $y=0(2 \mathrm{~m}<x<6$ $\mathrm{m})$ and as $u_{r}=-0.005 x+0.1 \mathrm{~m} / \mathrm{s}$ at $x=0(4 \mathrm{~m}<y<12 \mathrm{~m})$ approximately. If we assume the incompressibility of water current and the velocity field to be axisymmetric around the $z$ axis, $\frac{\partial u_{z}}{\partial z}=-\frac{1}{r} \frac{\partial}{\partial r}\left(r u_{r}\right) \approx 0.01 \sim 0.02 / \mathrm{s}$. The vertical component of water current, $u_{z}$, vanishes at $z=0$. Therefore, we can estimate the vertical component of water flow, $u_{z}$, as approximately 1 $\mathrm{mm} / \mathrm{s} \sim 2 \mathrm{~mm} / \mathrm{s}$ in the cylindrical shell region within radius $6 \mathrm{~m}<r<12$ $\mathrm{m}$. In this cylindrical shell region, there is down-flow from the surface by at least $u_{z}>1 \mathrm{~mm} / \mathrm{s}$ and oxygen dissolved naturally at the surface from the atmosphere may be directly supplied to the reservoir bottom.

In the aforementioned commercial shrimp aquaculture at the ABC Center of Karawang, Indonesia, it has been observed that shrimps gather immedi- 

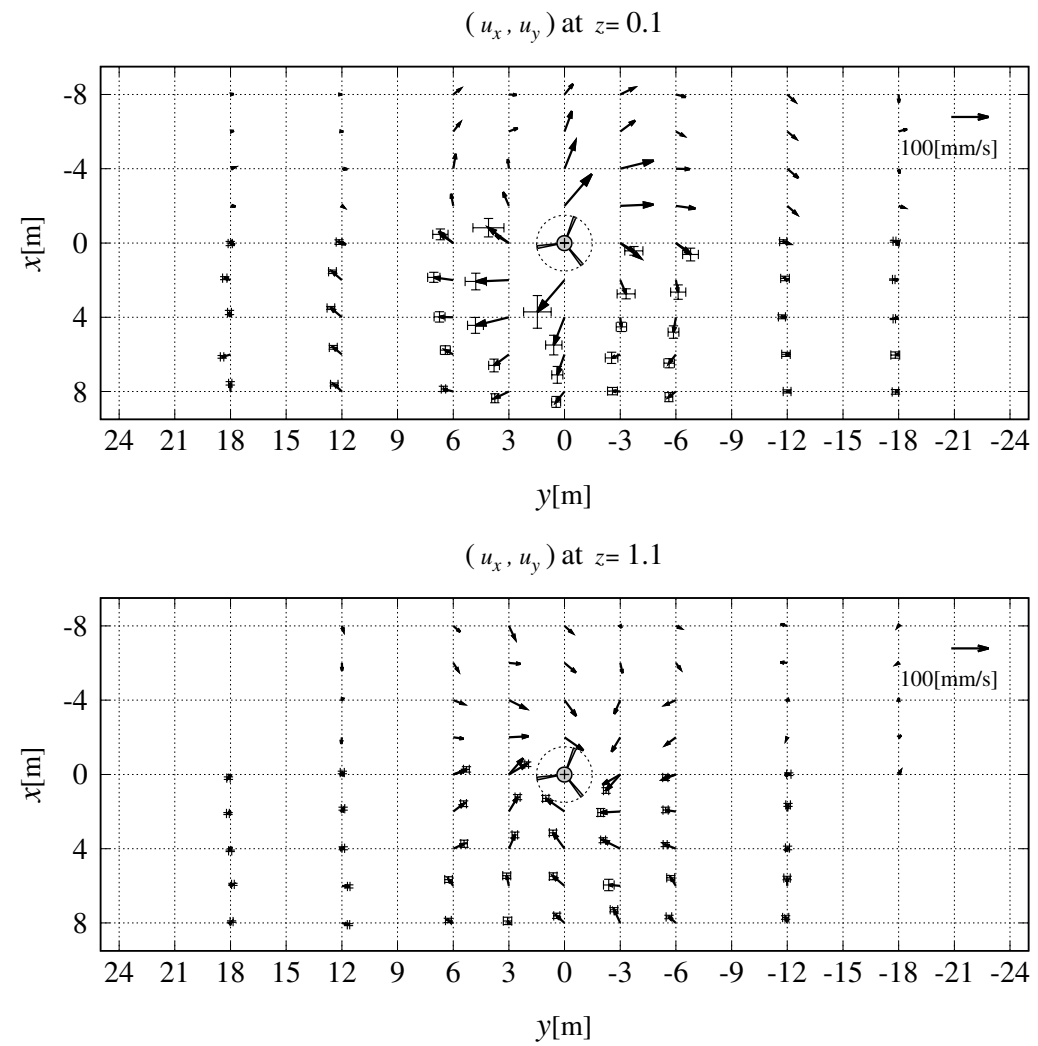

Figure 10: Velocity field of the water current induced by CWS. Measurements were taken at two horizontal planes, (upper) $z=0.1 \mathrm{~m}$ and (lower) $z=1.1 \mathrm{~m}$.

ately under CWS during early morning hours. This suggests that water circulation between the surface and the bottom resolves the DO shortage under the CWS. Additionally, a circle at the bottom with a radius of $7 \mathrm{~m}$ centered at the origin had horizontal water current over $15 \mathrm{~mm} / \mathrm{s}$. An optimal arrangement of aerators within a rectangular pond was discussed previously. For example, Peterson et al. (2001) performed a numerical simulation and recommended diagonal or parallel arrangement of paddle-wheel aerators to sustain a large horizontal circulation in a rectangular pond. Adopting the value of the radius as an indicator, one would optimally allocate the positivenegative rotating CWSs alternatively at distances of $14 \mathrm{~m}$ in a large pond.

Electric Power. - Finally, we should take into account the electric power consumed by both the aerators. In the present measurements, TA-55H and 


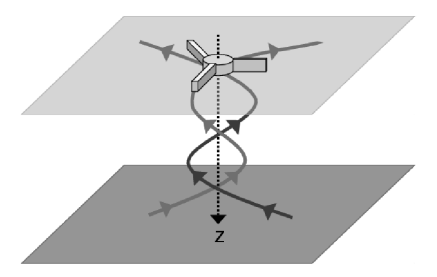

Figure 11: The expected spiral trajectories of fluid percels under CWS. The radial component of water current fields on the surface and bottom planes implies the upward flow under CWS and the circulation between the surface and bottom throughout the reservoir.

CWS worked at 630 Watts and 40 Watts, respectively. Thus, the paddlewheel aerator consumed over ten times the running cost of the centrifugalforce aerator. The kinetic energy of water current in the reservoir is, however, not largely different between them. The large difference between them can be attributed to the fact that most of the energy provided to TA-55H may be consumed in enhancing DO near the aerator, but CWS consumes electric energy to generate water current kinetic energy. From the previous measurement by Inagaki et al. (2017), the upward current under the CWS was estimated as $550 \mathrm{~m}^{3} / \mathrm{h}$. According to Brown et al. (2016), the flow rate of the CWS is less than that of paddle-wheel aerators, but water discharge per unit power input of the CWS is fairly efficient because of its lower energy consumption and of water exchange directly transfering between the surface and bottom.

\section{Conclusion}

In order to establish an optimal aeration system to maintain the DO value required for biological activity in water, we evaluated the water flows in a rectangular reservoir induced by a standard commercial Taiwanese paddlewheel aerator and a self-designed centrifugal water stirrer. The reservoir had a maximum depth of about $1.4 \mathrm{~m}$, and the measurements were taken using an electro-magnetic water current meter. Water velocity was found to be horizontally unidirectional for the paddle wheel aerator, and vertical circulation between the surface and the bottom induced by the paddle wheel aerator was smaller than that by the CWS aerator. The generated jet stream loses its kinetic energy according to water viscosity, and thus its contribution 
to the high DO transfer may be restricted only in the vicinity of the aerator downstream. The mean electric power consumed to operate the aerators was distinctly different between the two types of aerators, and most of the energy provided to the paddle wheel aerator were probably consumed in the enhancement of DO near the aerator, or otherwise simply consumed in generating intense splashing and large noise. The measurement of the DO field in the reservoir will be conducted in future work.

\section{Acknowledgement}

We wish to appreciate Mr Hosokawa and Mr Izu for their efforts to promote the research at the primary stage. This research is a product of research financially supported by the Kansai University Fund for Supporting Young Scholars 2016-2017, and ORDIST group fund 2018. The authors acknowledge Suita City Hall for kindly providing Katayama civic outdoor pool as an experimental reservoir. Finally, we would like to thank Editage for English language editing.

\section{References}

Boyd, C., 1998. Pond water aeration systems. Aquacultural Engineering 18, $9-40$.

Brown, T. W., Tucker, C. S., 2014. Pumping performance of a modifed commercial paddlewheel aerator for split-pond aquaculture systems. North American Journal of Aquaculture 76, 72-78.

Brown, T. W., Tucker, C. S., Rutland, B. L., 2016. Performance evaluation of four different methods for circulating water in commercial-scale, split-pond aquaculture systems. Aquacultural Engineering 70, 33-41.

Cancino, B., 2004. Design of high efficiency surface aerators: Part 2. rating of surface aerator rotors. Aquacultural Engineering 31, 99-115.

Cancino, B., Roth, P., Reuß, M., 2004. Design of high efficiency surface aerators: Part 1. development of new rotors for surface aerators. Aquacultural Engineering 31, 83-98.

Colt, J., Orwicz, C., 1991. Aeration in intensive culture. Aquaculture and Water Quality 3, 198-269. 
Gillet, R., 2008. Global study of shrimp fisheries. FAO fisheries technical paper 475 .

Huggins, D. L., Piedrahita, R. H., Rumsey, T., 2004. Analysis of sediment transport modeling using computational fluid dynamics (cfd) for aquaculture raceways. Aquacultural Engineering 31, 277-293.

Inaba, H., 2005. Floating type energy saving water cleaning apparatus. Japanese Patent (3360075).

Inagaki, T., Nakamura, C., Itano, T., Sugihara-Seki, M., Hyodo, J., 2017. Water circulation induced by a centrifugal water streamer in rectangular reservoirs. Transactions of the Japan Society of Mechanical Engineers 83.

Itano, T., Inagaki, T., Nakamura, C., Hashimoto, R., Negoro, N., Honda, S., Hyodo, J., 2018. Water circulation driven by a paddle-wheel aerator in rectangular reservoir. Science and Technology Reports of Kansai University $60,15-22$.

Lawson, T. B., 1997. Fundamentals of aquacultural engineering. CBS publishers, New Delhi.

Moulick, S., Mal, B., Bandyopadhyay, S., 2002. Prediction of aeration performance of paddle wheel aerators. Aquacultural Engineering 25, 271-237.

Murai, Y., 2007. Ebi to Nihonjin II. : kurashi no naka no gurobaru-ka (in Japanese). Iwanami Shoten.

Peterson, E. L., Harris, J. A., Wadhwa, L. C., 2000. Cfd modelling pond dynamic processes. Aquacultural Engineering 23, 61-93.

Peterson, E. L., Wadhwa, L. C., Harris, J. A., 2001. Arrangement of aerators in an intensive shrimp growout pond having a rectangular shape. Aquacultural Engineering 25, 51-65.

Pfeiffer, T., Lawson, T., Church, G., 2007. Engineering considerations for water circulation in crawfish ponds with paddlewheel aerators. Aquacultural Engineering 36, 239-249.

Yamayoshi, N., Kitazawa, D., Konno, A., Chiba, K., 2008. Numerical simulation of the current field in the small cultivation pond with the effect of paddle wheel. SEISAN KENKYU 60, 55-58. 